\title{
Recovery countermeasure features that may preclude MCDA based decisions
}

\author{
K.G. Andersson* \\ DTU - Technical University of Denmark, Roskilde, Denmark.
}

\begin{abstract}
Multi Criteria Decision Analysis (MCDA) with stakeholder participation has been suggested as a way to reach mutually acceptable and robust decisions regarding methods and strategies for recovery of inhabited land areas and food production systems severely contaminated by an airborne radioactive plume from a severe nuclear power plant accident. The paper describes a new approach to help ensuring that important issues are not overlooked when decisions are based on MCDA.
\end{abstract}

Keywords: accident / nuclear / cost-benefit analysis / contamination / environmental / recovery / radiation protection

\section{Introduction}

Nuclear and radiological accident scenarios that significantly affect large areas of land are generally highly challenging and complex to address with the objective of optimized restoration and recovery of normal lifestyles to the extent possible. This is because each potentially applicable restoration option (countermeasure) is associated with a large number of case specific features or criteria, which can have positive and/or negative effects on the implementation outcome of that countermeasure. Such criteria could for example comprise the health effect or radiological impact (e.g., change in dose reduction to the population, incremental dose to countermeasure implementers), direct costs of the implementation (e.g., worker wages, equipment, consumables, transport, treatment and storage of any waste produced, and other monetary influences), environmental impact (e.g., risk of pollution of groundwater, impact on soil fertility, risk of erosion), legal impact (conflicts between the outcome or processes of the intervention and current legislation in the area), social impact (e.g., restrictions on further use of land and amenities, loss of income, communication needs) and ethical impact (e.g., equity in countermeasure implementation, and public consent) (Nisbet et al., 2010). Only few such criteria can, within some uncertainty bounds, be reasonably quantified in units (e.g., monetary) in a way that permits partial analysis of the relative desirability of each option in the specific case.

To facilitate the process of decision making, a number of scholars (see, e.g. Mustajoki et al., 2007) suggested to employ a systematic computer-based approach to examine the options, their features and the entire trade-off process involved in optimized decision making throughout all the facilitated

\footnotetext{
*Corresponding author: kgan@dtu.dk
}

interactions and discussions with stakeholders (representatives of individuals, groups or parties who are somehow affected by the decisions to be made, or in other ways have a share in them). MCDA (multiple-criteria decision analysis) is a computer-based method to evaluate multiple conflicting criteria in decision making (see more on its application in Hiete et al., 2010). In MCDA, a first step is to establish the aim for the particular scenario in question (context). Then the options that may potentially be used to reach the aim should be identified (intervention options). The next step would be to identify criteria for assessing the consequences of the individual options. A number of examples of such criteria were given above, in the first paragraph. Once the criteria are identified one needs to evaluate the consequences of the possible impact under each criterion and on this background to score the intervention options on the criteria. Each of these scores would often be based on a multitude of influencing factors, and it will, therefore, not be easy to have a full overview. Finally, weights are assigned to each of the criteria to express the relative importance of these. This process is challenging as weights are here given to more or less general features like "health effect", "direct costs", "social impact" and "environmental impact", perhaps without the possibility of keeping it very clear in mind exactly which issues lay beneath in the specific case.

MCDA has its weaknesses (or challenges), but when a decision must be taken as to which intervention options should be implemented, following this type of procedure can in principle encompass evaluation of all factors deemed to be of importance.

\section{Problem description}

The decision process using MCDA is highly sensitive to particularly the relative scores given to the intervention options on each criterion, as well as to the weights assigned to each 


\begin{tabular}{|c|c|}
\hline \multicolumn{2}{|r|}{ Deep ploughing } \\
\hline Objective & $\begin{array}{l}\text { To reduce inhalation and external gamma and beta doses from } \\
\text { contamination in outdoor areas covered in grass or soil within } \\
\text { inhabited areas. }\end{array}$ \\
\hline Health & $\begin{array}{l}\text { First assess that the overall health risk in the area can be brought to a } \\
\text { level that permits people to stay there. } \\
\text { Poor beneficial effect likely if soil is very sandy or dry. } \\
\text { Requires thorough instructions for operator to optimize positive health } \\
\text { effect. }\end{array}$ \\
\hline Direct costs & - \\
\hline Environment & $\begin{array}{l}\text { Loss of biodiversity in the treated area. Loss of fertility in the treated } \\
\text { area. } \\
\text { Assess and consider groundwater level in advance. }\end{array}$ \\
\hline Legality & $\begin{array}{l}\text { Owners' / society's permission. } \\
\text { Subsequent restrictions on tilling of treated land. }\end{array}$ \\
\hline Social & $\begin{array}{l}\text { The option does not remove the contamination from the area. It makes } \\
\text { it virtually impossible to ever remove the contamination if desired later. } \\
\text { Adverse aesthetic effect }\end{array}$ \\
\hline Ethical & Owners' / society's consent. \\
\hline
\end{tabular}

Fig. 1. Example of datasheet from the new recovery handbook addendum, describing particularly problematic countermeasure features that it is deemed may preclude MCDA based decisions.

criterion. If for example all scores have to be given on a scale from 0 to 100 , a truly undesired (potentially disastrous) environmental feature of an option would result in a very low score (perhaps even 0) on this criterion (although other environmental features may also influence the overall score of the criterion). At the stage of weighting the criteria, it might be that due to priority focus on, e.g., solving the health problem thoroughly and taking good care of the social impact, the environmental criterion would receive a relatively low weighting, which could mean that for example an alternative option with criteria where there were perhaps no scores lower than 10 (no potential disasters) would come out of the analysis with less total "desirability" (lower overall weighted score).

The example above highlights that although the MCDA process seemingly offers great transparency and provides a result that involved stakeholders will likely see as a "best compromise" that takes into account all important factors, and, thus, facilitates consensus, there is a risk that important aspects may be hidden in the process, since the assumption that scores can unproblematically be added and weighted against each other to provide the most desirable solution will not necessarily be valid in all cases.

\section{Results-Proposed problem solution}

To avoid problems being overlooked in the MCDA process it is thus very important that the facilitator of the decisionmaking process (or a person acting in the process as co-facilitator) has in-depth knowledge of the intervention options and their potential impacts with respect to the different criteria used in the analysis. However, the deep expert knowledge on all countermeasure options will often not be available in national fora making the decisions. In order to avoid that potentially crucial features are lost track of in the processing of criteria scores and weights, it can be useful to provide an overview for the decision-makers of such features that may at least under some conditions lead to not readily reversible severe repercussions. Therefore new work has been done to pinpoint, in an "easy-overview" format, features of each of the recovery options in the European Handbooks, which could be perceived as particularly problematic and lead to (not easily reversible) undesired effects.

The intention is that these short texts could be reviewed together with the overall weighted scores produced by MCDA to ensure nothing important is forgotten when the decision is taken. However, for the MCDA process itself, it is important to look at all the features described for each countermeasure in the relevant European recovery handbook. The new short texts are not suitable either for, e.g., countermeasure implementers, who need a much wider range of considerations, some of which are treated in the European recovery handbooks. It is assumed that countermeasures are applied in reasonable compliance with the handbook recommendations.

The criteria selected for this particular purpose relate to health, direct costs, environment, legality, social and ethical concerns. The datasheet in Figure 1 below shows an example of some countermeasure features that might be particularly problematic to consider in decision-making (in this case for "deep ploughing"). The new recovery handbook addendum (Andersson, 2019) contains 88 datasheets of this type, relating to all the recovery countermeasures for the inhabited areas and food production. 


\section{Conclusions}

Stakeholder guided MCDA is a method for gaining consensus about decisions regarding recovery options. However, the list of features that should be considered as input to the decision process is very long, and it can be difficult to keep track of all concerns of importance. Therefore, the addendum to the European recovery handbooks described in this paper has been created to aid the decision-making process and ensure that important issues are not overlooked.

Acknowledgement. The work described in this paper was conducted within the CONFIDENCE project which was part of the CONCERT project. This project has received funding from the Euratom research and training programme 20142018 under grant agreement No. 662287.

Disclaimer (Art. 29.5 GA). This publication reflects only the author's view. Responsibility for the information and views expressed therein lies entirely with the authors. The European
Commission is not responsible for any use that may be made of the information it contains.

\section{References}

Andersson KG. 2019. Potentially overruling countermeasure features in decision making based on MCDA, CONFIDENCE WP6 project internal report.

Hiete M, Bertsch V, Comes T, Schultmann F, Raskob W. 2010. Evaluation strategies for nuclear and radiological emergency and post-accident management. Radioprotection 45(5): S133-S147.

Mustajoki J, Hämäläinen RP, Sinkko K. 2007. Interactive computer support in decision conferencing: two cases on offsite nuclear emergency management. Decis. Support Syst. 42: 2247-2260.

Nisbet AF, Andersson KG, Duranova T. 2010. Demonstration of generic handbooks for assisting in the management of contaminated food production systems and inhabited areas in Europe. Radioprotection 45(5): S243-S249.

Cite this article as: Andersson KG. 2020. Recovery countermeasure features that may preclude MCDA based decisions. Radioprotection 55 (HS1): S205-S207 